\title{
APPrender a leer y escribir: aplicaciones para el aprendizaje de la lectoescritura
}

\section{Learn to read and write: app for the literacy learning}

Raquel Gómez-Díaz, Araceli García-Rodríguez, José Antonio Cordón-García

Departamento de Biblioteconomía y Documentación, Facultad de Traducción y Documentación,Universidad de Salamanca, Spain \{rgomez, araceli, jcordon\}@usal.es

Resumen

El aumento de las tabletas y de las aplicaciones educativas destinadas al público infantil hace necesario, además de analizar las ventajas e inconvenientes de su utilización en el aula, tener un mayor conocimiento sobre las propias aplicaciones, sus características, modelos de negocio, etc. El artículo se centra en las apps destinadas al aprendizaje de la lectoescritura, y ofrece, además de una tipología de las mismas, y una selección de algunas de ellas. Finalmente se incluye un listado de fuentes que ayudará a los maestros en la tarea de selección de las aplicaciones más adecuadas para las necesidades de los alumnos.

\section{Palabras Clave:}

Aprendizaje de lectoescritura; app educativas; tabletas en el aula.

\section{Abstract}

Increasing tablets and educational apps aimed at kids made necessary, besides analyzing the advantages and disadvantages of their use in the classroom, have a better understanding of the applications themselves, their characteristics, business models, etc. The article focuses on the apps intended for literacy learning, and offers, along with a typology, and a selection of some of them. Finally, it includes a list of sources that will help teachers in the task of selecting the most suitable for the needs of pupils' applications.

\section{Keywords:}

Learn to read; learn to write; educational app; tablets in the school. 


\section{Introducción}

El aumento del uso de tabletas, la gran variedad de aplicaciones educativas, su uso creciente por parte de los llamados "nativos digitales" (Presnsky, 2001), y su integración en el aula suponen un reto que debe hacer reflexionar a los docentes sobre las nuevas posibilidades que ofrecen en el aula $y$ llevarles a integrar estas herramientas en su metodología diaria de trabajo.

Es evidente que la simple utilización de tabletas en el aula no aumenta la calidad educativa (Martín, Silvia, 2011), que no basta con la mera motivación de los alumnos por el uso de los dispositivos para justificar su presencia, si esta utilización no va acompañada de una integración adecuada en el proceso de aprendizaje, de una correcta selección de aquellos productos que permitan realmente alcanzar las competencias deseadas y de la formación de los docentes en el uso de dispositivos y aplicaciones. Si bien las tabletas crean nuevas oportunidades de aprendizaje, no pueden utilizase de cualquier manera si se quiere asegurar el desarrollo de competencias (Northrop, 2013) ya que en ocasiones parece que la tecnología distrae del objetivo principal (Chiong, Ree, Takeuchi \& Erickson, 2012). Tal y como afirmaba Francesco Tonucci en una entrevista realizada en 2013 en el Tiching $\mathrm{B} \log$

\footnotetext{
"Las tecnologías son un gran invento pero no hay que olvidar que son un instrumento que solo vale si el que lo utiliza es bueno [...] No serán las
}

tecnologías las que mejoraran las escuelas, ni tampoco las leyes. Serán los buenos maestros".

Por tanto, la actitud de los profesores y su formación es decisiva a la hora de aprovechar las ventajas tecnológicas y transformar la pedagogía. Cuanto más abiertos se muestren ante los cambios, mayor sea su capacidad de adaptación, más adecuada su competencia digital y mediática, más profundo y rápido será el cambio de cultura educativa y más efectiva será su apropiación de los nuevos recursos tecnológicos (Perspectivas 2014).

Teniendo en cuenta estas cuestiones, el artículo pretende:

- Señalar las ventajas e inconvenientes de la utilización de tabletas y aplicaciones en el aula.

- Ofrecer una clasificación de las aplicaciones de lectoescritura, así como una serie de ejemplos que permitirán a los docentes aproximarse a las aplicaciones relativas a esta competencia.

- Mostrar algunas de las características de las aplicaciones de lectoescritura.

- Enunciar algunas fuentes y recursos útiles para la selección de aplicaciones educativas.

A fin de contextualizar el tema el segundo apartado se centra en el estudio del uso de tabletas y aplicaciones en el aula, sus ventajas e inconvenientes, para pasar posteriormente a analizar las características y contenidos de las aplicaciones educativas en general y de 
las de lectoescritura en particular. El artículo se completa con las conclusiones finales, las referencias bibliográficas y dos anexos: uno relativo a las fuentes y recursos sobre aplicaciones educativas (anexo 1) y otro que

\section{Metodología}

La búsqueda de documentación se centró por un lado en la utilización de las aplicaciones de lectoescritura en los colegios y por otro en el contexto en el que se produce la formación. Una de las principales dificultades es la escasez de literatura científica sobre el tema. A pesar de realizar búsquedas bibliográficas en la base de datos especializada en educación ERIC y en la generales del CSIC, WOS y Scopus, así como en Google Scholar, Los resultados son escasos y ninguno de ellos relativo a experiencias realizadas en el contexto español. Es cierto que existe bibliografía sobre las aplicaciones educativas en general, pero no sobre las de lectoescritura en particular. Esta carencia se puede suplir con las experiencias que aparecen reflejadas en distintos blogs y portales de carácter educativo como los mencionados en el anexo 1.

En lo relativo al contexto se procedió a la consulta de diferentes informes sobre la penetración de las tabletas y las aplicaciones educativas en el mercado como Perspectivas recoge la descripción detallada de diferentes aplicaciones clasificadas según las categorías descritas previamente en el artículo (Anexo 2)

(2014), Metainvestigación Dim-Edu (20132014), el Proyecto e-book y educación. Dedos: tabletas digitales en el aula (2010-2011) o Sistema Uno de la Fundación Santillana, entre otros, que pretenden explorar las posibilidades educativas de las tabletas (http://www.monografias.com/trabajos93/ uso-tablets-educacion/uso-tablets-educacion. shtml).

Para seleccionar las apps más adecuadas se siguieron los distintos niveles de lectoescritura (presilábico, silábico, silábico-alfabético y alfabético) definidos por Ferreiro y Teberosky (1979). Fue necesaria la consulta de distintas fuentes como son los blogs y recomendadores (Frikids, Smartappsforkids, Aplicaditos, La souris grise, Pequetablet, Literaturas Exploratorias, Bestappsfokids, Proyecto Guappis, etc.).

Finalmente se procedió a la Descarga y testeo de las aplicaciones y a la elaboración del listado de aplicaciones recomendadas.

\section{El uso de tablets y aplicaciones en el aula}


Según el estudio Perspectivas 2014 los Tal como se afirma en la investigación entornos digitales, redes sociales, juegos DIM-EDU (http://peremarques.net/ educativos en red, etc. serán una realidad en tabletasportada.htm) sobre el uso educativo las aulas en el 2020. Este informe señala que de las tabletas realizada entre 2012 y 2013, la cronología de implantación será en cuatro estos dispositivos cuentan con una serie de etapas tal y como se muestra en la Infografía. ventajas entre las que se destacan:

Integración de los servicios digitales en el • Acceso a múltiples fuentes de información. aula. Los alumnos no dependen solo del libro

\section{LAS AULAS ESPAÑOLAS DEL SIGLO XXI: la integración de los servicios digitales}

Los expertos en innovación tecnológica educativa y pedagogía prevén cómo se integrarán los servicios digitales educativos

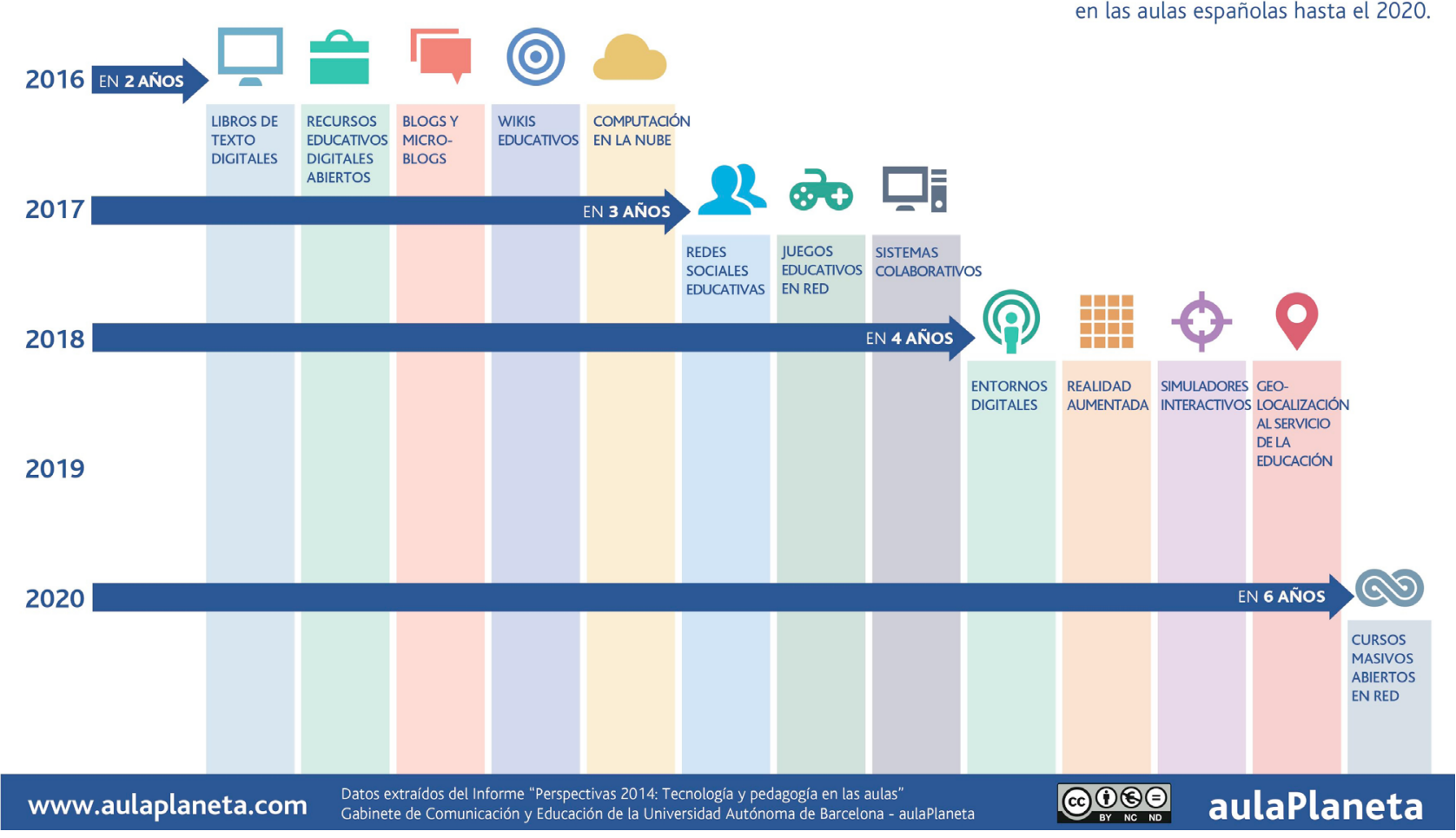

Fig. 1. Infografía. Integración de los servicios digitales en el aula

Fuente: Perspectivas 2014: Tecnología y Pedagogía en las aulas. http://www.aulaplaneta.com/2015/02/09/noticias-sobre-educacion/la-escuela-digital-sera-una-realidad-en-2018-infografia/\#sthash.w4KpZavL.dpuf

La infografía muestra que en un futuro, más o menos próximo, las tecnologías digitales estarán cada vez más presentes y eso dará lugar a una nueva forma de enseñanzaaprendizaje, más activa y participativa en la que las tabletas pueden llegar a tener un papel destacado. de texto y de la explicación del profesor.

- Colaboración y apoyo. Los usuarios se pueden conectar con compañeros y profesores y acceder a aplicaciones y entornos colaborativos, compartir.

- Portabilidad. Su tamaño y peso facilitan su uso en cualquier entorno.

- Multifuncionalidad. En una tableta los 
alumnos tienen todo lo necesarios para hacer diferentes actividades.

- Aprendizaje autónomo y creatividad. Las tabletas permiten a los alumnos ser autónomos en la búsqueda de información y uso de herramientas.

- Aplicación de nuevas metodologías didácticas que permiten una enseñanza basada en el estudiante.

- Motivación e implicación. Se constata que los alumnos están más motivados cuando utilizan este tipo de dispositivos.

- Eficacia y eficiencia.

- Mejora en los aprendizajes y el rendimiento académico

- Comodidad. Los alumnos se sienten cómodos en el mundo de los dispositivos móviles de hecho, 7 de cada 10 niños menores de 12 años utilizan un dispositivo móvil; el 10\% de los menores de un año, el $39 \%$ de los menores de 4 y el $50 \%$ en los niños hasta 8 años ${ }^{1}$.

- Difusión social de las competencias digitales. En ocasiones los padres se interesan por las tabletas y las aplicaciones al comprobar el uso que hacen de ellas sus hijos.

Por tanto, los dispositivos móviles y las aplicaciones diseñadas para ellos, pueden aportar un valor añadido en el proceso de enseñanza-aprendizaje, favoreciendo el desarrollo de determinadas destrezas y competencias al tiempo que constituyen una motivación extra para los alumnos.

Pero no se puede obviar que su uso plantea limitaciones y problemas, tanto técnicos y de manejo como derivados de su uso pedagógico. Según la mencionada investigación las imitaciones técnicas más destacables son: La conexión a Internet puede ser lenta o no funcionar. Además, en algunos casos los filtros de acceso Internet del centro pueden impedir el acceso a contenidos necesarios.

- Averías en los equipos.

- Problemas con el software.

- Incompatibilidad entre dispositivos.

- Incomodidad del teclado digital.

- La necesidad de introducir una cuenta personal para poder descargar las apps

En cuanto a los problemas derivados del uso pedagógico, se pueden destacar:

- La escasez de contenidos educativos en castellano. Afortunadamente los fabricantes tecnológicos son conscientes de estas carencias y están poniendo en marcha programas para entornos educativos como es el caso de Samsung School presentada en octubre de $2014^{2}$.

- La dependencia de la tableta, que pueden producir alteraciones en la dinámica de las clases cuando los alumnos se olvidan la tableta, se acaba la batería, no actualizan las aplicaciones, etc.

- La falta de tiempo de los profesores para buscar y desarrollar recursos.

- Distracciones con otras aplicaciones lúdicas, redes sociales, etc.

A esto hay que añadir que no todos los colegios están dotados con las infraestructuras y los presupuestos necesarios para llevar a cabo con buenas garantías la implantación de la tableta en el aula, ni todos los docentes están 
preparados para ello.

Es cierto que el futuro de las tabletas en el aula no está definido todavía, pero no se puede olvidar que los niños conviven con ellas. Como señala Silvia Martín (2011) el dilema no está en ni el uso de las tabletas ni sus aplicaciones, sino en cómo se usan. Pueden ser de gran ayuda siempre que se entienda que no son un objetivo, sino el medio para conseguir un fin: la mejora del proceso de enseñanza-aprendizaje. Su uso no sirve de nada si no está apoyado en buenas actividades didácticas o si el profesorado no está formado para usarlas correctamente y aprovechar todas sus potencialidades.

Finalmente es importante señalar que no todas las apps que entran dentro del grupo de las denominadas educativas lo son, por lo que un requisito fundamental es conocer sus características, funciones y valores pedagógicos (Rojas, G.; Celaya, J; Vázquez, J.A, 2014, p. 53).

\section{Metodología}

El aumento del uso de las tabletas ha producido el crecimiento de aplicaciones de todo tipo. Según los últimos datos disponibles las educativas gozan de un gran prestigio y una importante cuota de mercado. En el caso de Apple, ocupan el tercer lugar entre las más populares, solo por detrás de las del sector de negocios y videojuegos (marzo 2015 http://www.statista.com/statistics/270291/ popular-categories-in-the-app-store/), y el primer puesto para las creadas para Android (abril 2015 http://www.appbrain.com/stats/ android-market-app-categories).

Ya en abril del año 2014 el portal Universia (http://noticias.universia.es/ciencia-nn-tt/ noticia/2014/04/07/1093782/ya-existenmas-80-000-aplicaciones-educativas.html) estimaba que existían más de 80.000 aplicaciones educativas de todas las materias, edades, idiomas, etc. y de hecho, después del mundo de los videojuegos, la educación es el sector con el mayor número de aplicaciones móviles, una cifra que sin duda alguna seguirá en aumento.

Si nos circunscribirnos a los usuarios infantiles, según los datos del Instituto Nacional de Estadística ${ }^{3}$, en 3 de cada 10 hogares los niños utilizan la tableta (la mayoría tiene de 7 a 12 años) lo que supone aproximadamente el $77 \%$ de los hogares con niños. Además, tal como se afirma en el último estudio sobre Menores de Edad y Conectividad Móvil en España (2014), el acceso a las TIC se produce a edades cada vez más tempranas; ya desde los 2-3 años acceden de forma habitual a los dispositivos de sus padres y manejan diferentes aplicaciones para pintar o colorear, ver series de televisión, vídeos y fotografías, etc.

Estos mismos datos vienen confirmados por el último Informe Tab (2014), según el cual aunque las aplicaciones más populares en las 
familias con hijos siguen siendo los juegos, las educativas representan el $36 \%$ de todas las descargadas en España y son cada vez más los profesores y alumnos que las utilizan para hacer todo tipo de tareas, tanto en el aula como en el hogar.

La importancia que están cobrado las apps educativas viene corroborada por la creación de secciones específicas en por parte de de Apple y Google.

Apple ha hecho una apuesta importante por el uso del iPad creando una división específica para educación (https://www.apple.com/es/ education/) y las aplicaciones educativas, que suponen el 80\% (75.000 productos incluyendo materiales para alumnos y profesores) de todas las desarrolladas para este sistema.

Google también cuenta con una extensión educativa de Play Store (https://www. google.com/edu/index.html) para profesores de primaria y secundaria en Estados Unidos. De momento no existe la posibilidad de crear cuentas específicas para estudiantes y profesores ni permite a los desarrolladores que venden en Play Store establecer funcionalidades y precios distintos a los que se ofrecen en el marketplace.

Conviene hacer hincapié en que para el mundo educativo el sistema operativo preferido por los usuarios como por los profesionales es iOS por la calidad de sus productos, aunque cada vez es más habitual que los desarrolladores lancen versiones de las mismas aplicaciones también en Android. En muchos casos, aunque se trate de la misma aplicación, esta puede tener características e incluso precios diferentes en función del sistema operativo para el que haya sido diseñada.

En lo relativo a la adquisición, a diferencia de otras categorías de aplicaciones, en muchas ocasiones gratuitas, son relativamente pocas las aplicaciones educativas que se pueden adquirir sin coste. Existen varias opciones, en muchos casos combinables, entre los que podemos destacar ${ }^{4}$ :

- Versión gratuita. Con acceso ilimitado a todos los contenidos.

- Versión gratuita/ lite. Este modelo consiste en ofrecer algún producto o contenido de manera gratuita mientras que para tener acceso a otra parte de los contenidos hay que descargarse la versión de pago.

- Versión gratuita con publicidad. No es una modalidad recomendada para niños y menos aún para el uso en el aula.

- Versión gratuita con compras integradas (in app purchase). La descarga de la app suele ser gratuita o con un precio bastante asequible. Los primeros contenidos, juegos, niveles, etc., van incluidos con la descarga y una vez utilizados el usuario puede pagar por el resto a través de micropagos.

- Versión de pago. En la mayoría de los casos permiten el acceso total a todos los contenidos, pero podemos encontrarnos con algunos ejemplos en los que se incluyen también compras integradas.

- Suscripción. El modelo de suscripción no es habitual en este tipo de app aunque hay algunos ejemplos en el caso de juegos 
educativos.

- Bundles o paquetes de aplicaciones, normalmente con el mismo tipo de contenidos.

- Compras especiales para centros educativos. Es el caso de Apple que dispone del programa PCV, Compras por Volumen en el Sector Educativo. A través de él es posible comprar apps a menor precio. Los desarrolladores pueden activar precios especiales dentro del programa y los centros recibir un descuentos del $50 \%$ cuando compran 20 o más unidades.

Con el PCV los centros educativos tienen la propiedad y el control del contenido a través de soluciones de gestión de dispositivos móviles (MDM) ${ }^{5}$ o mediante códigos canjeables y los usuarios pueden disfrutar del acceso inalámbrico a las apps (https://www.apple. com/es/education/docs/VPP_Education_ Guide_ABC_Aug14.pdf)

Por lo que se refiere a las etapas educativas según el estudio iLearn II Analisis of Education Category of Apple's App Store (Shuler, 2012) el número de aplicaciones destinadas a la etapa pre-escolar, entre 0

Chart 2: Target Age, Overall Vs Top 25
Percent of top-selling apps which target:

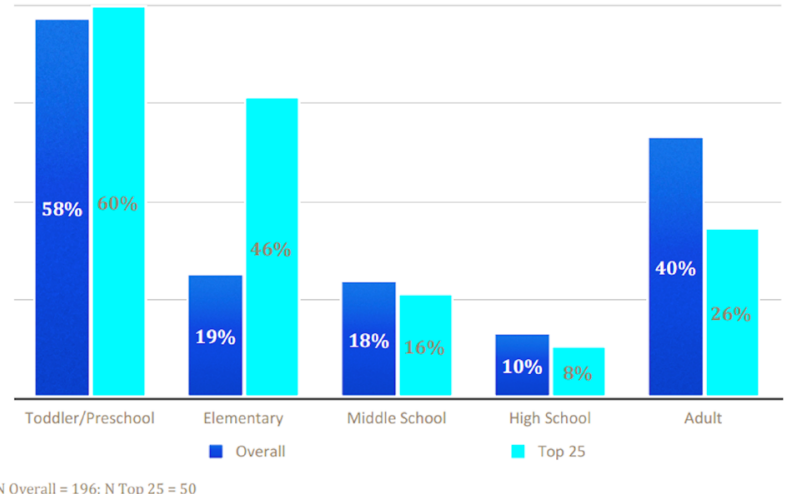

Fig. 2. Aplicaciones por nivel escolar en Apps Store Fuente: (Shuler, 2012) y 5 años es el más elevado, seguido de las destinados a la educación infantil y primaria y en tercer lugar la secundaria.

En lo relativo a los contenidos, existen productos para prácticamente cualquier tarea, por lo que es importante tener una idea clara de su clasificación.

Para ello, se pueden elegir criterios como la etapa educativa, los destinatarios (alumnos,
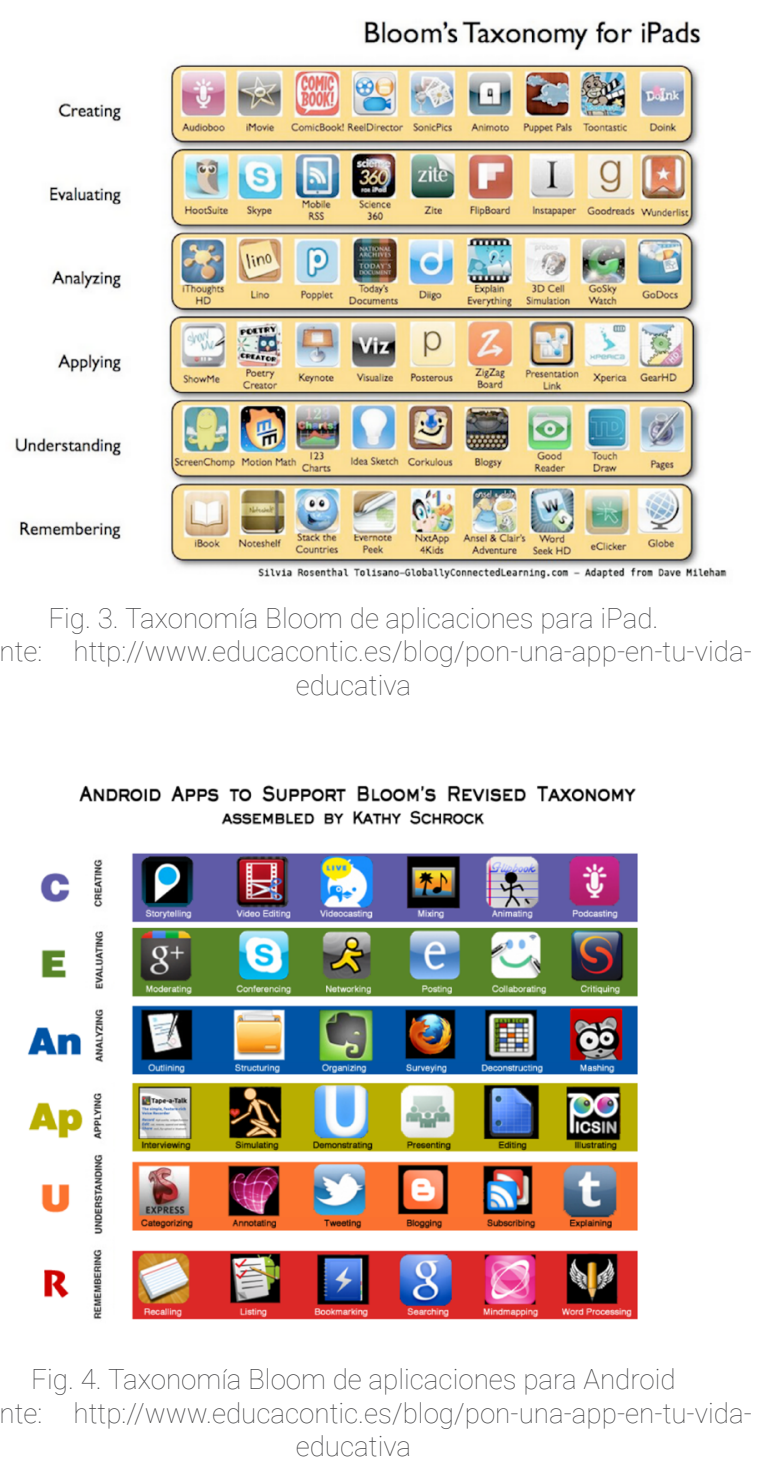

profesores, padres) o seguir la taxonomía de Bloom en la que se tiene en cuenta el tipo de competencias.

Sin embargo, aceptando la utilidad de esta taxonomía, se propone una clasificación 
basada en las materias troncales establecidas por la LOMCE para primaria y secundaria, siempre teniendo en cuenta que una app de la misma materia puede ser utilizada para trabajar diferentes competencias y que también existen productos para el resto de materias específicas, religión, educación física, educación plástica y visual, música, etc.

Dentro de esta variedad de contenidos, tal como afirman Guillem y Celaya (2014) existen dos tendencias fundamentales en las

\begin{tabular}{|c|c|}
\hline TIPO & CATEGORÍAS \\
\hline Instrumentales $^{6}$ & $\begin{array}{l}\text { Calendarios, notas, calculadora, diccionario, evaluación, mapas, } \\
\text { atlas, creación de vídeos... }\end{array}$ \\
\hline $\begin{array}{l}\text { Lengua castellana y } \\
\text { literatura }\end{array}$ & $\begin{array}{l}\text { Lectoescritura, ortografía, libros app, vocabularios, gramática, } \\
\text { géneros literarios, storytelling, aplicaciones de lectura... }\end{array}$ \\
\hline Matemáticas & Cálculo, algebra, geometría, resolución de problemas... \\
\hline Ciencias sociales & $\begin{array}{l}\text { Historia, arte, civilizaciones, geografía física, geografía política, } \\
\text { económica, demográfica. }\end{array}$ \\
\hline $\begin{array}{l}\text { Ciencias de la } \\
\text { naturaleza }\end{array}$ & $\begin{array}{l}\text { Ciencias naturales, biología, física y química, astronomía, } \\
\text { anatomía, geología, etc. }\end{array}$ \\
\hline Lengua extranjera & $\begin{array}{l}\text { Vocabularios, aprendizaje de un idioma, gramática, diccionarios, } \\
\text { fonética, libros en lenguas extranjeras... }\end{array}$ \\
\hline
\end{tabular}

Tabla 1 Tipos de aplicaciones educativas en función de la materia. Fuente: elaboración propia.

metodologías: gamificación y personalización. la gamificación (Guillem, Celaya, 2014). La gamificación hace referencia a la inclusión La personalización pretende que el niño se de mecánicas de juego en aspectos y contextos convierta en un elemento activo, que tenga que no tienen que ver con el juego. El juego la posibilidad de elegir entre tipos de letras, es una metodología natural, una herramienta idiomas, nivel de dificultad, perfiles, etc. 


\section{Apps para el aprendizaje de la lectoescritura}

El aprendizaje de la lectoescritura es uno de los más trascendentales en el desarrollo de un niño y enseñar a leer y escribir uno de los retos más importantes de todas las escuelas. No podemos olvidar que es una habilidad que está vinculada a la adquisición de otros aprendizajes y relacionada con el desarrollo madurativo del niño. Para ello, los maestros cuentan actualmente con recursos de todo tipo entre los cuales están empezando a destacar las aplicaciones para tabletas.

En los últimos años han ido apareciendo gran cantidad de aplicaciones de este tipo con las que los niños pueden aprender a leer y escribir de forma sencilla, divertida e interactiva, a desarrollar habilidades cognitivas y de comunicación, adquirir vocabularios, ejercitar la memoria, etc. permitiendo a los más pequeños iniciarse en la lectura y potenciar el proceso de comprensión lectora de una forma lúdica.

Conviene sin embargo no olvidar que hay que comprobar que los contenidos están adaptados a la capacidad y edad madurativa del niño y que el adulto debe acompañar el proceso de aprendizaje, ayudar con las posibles dificultades y corregir errores. La tableta puede sustituir a la antigua cartilla o al cuaderno de caligrafía, pero no al maestro que enseña, corrige y anima al niño en el aprendizaje.

Dentro de las aplicaciones de lectoescritura podemos encontrarnos con algunas diseñadas específicamente para este cometido, pero también con otras que sin estar pensadas inicialmente para ello, pueden ser utilizadas con este fin. La siguiente figura muestra una posible clasificación.

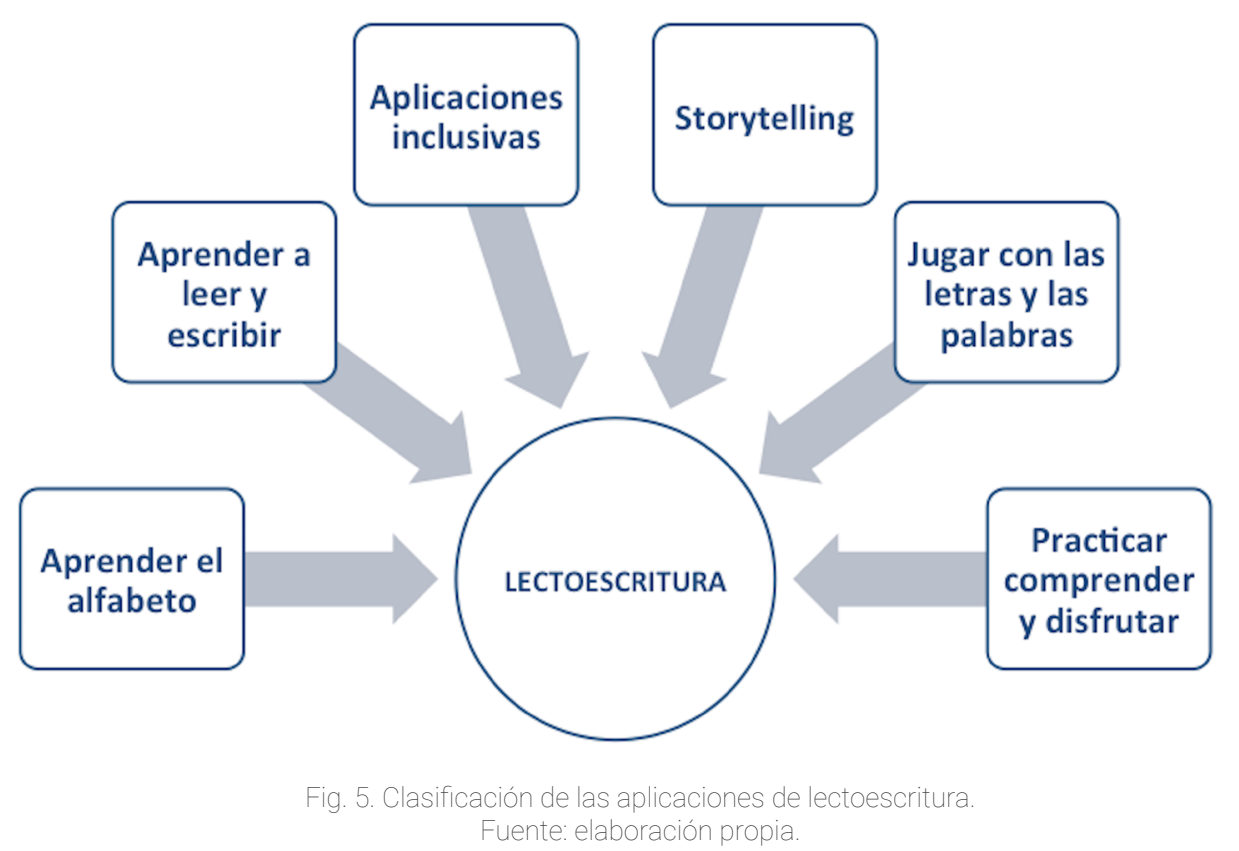




\subsection{Aprender el alfabeto}

Este tipo de aplicación son las más básicas y están dirigidas al conocimiento del alfabético, su fonética, e incluso la grafía, por parte de los más pequeños.

La mayoría están desarrolladas siguiendo la metodología Montessori, según la cual los niños necesitan relacionar cada letra con su fonema para aprender a leer, incluyen

\subsection{Aprender a leer y escribir}

Se incluyen en este caso aquellas aplicaciones centradas en dos tipos de aprendizajes.

- Letras, sílabas y composición de palabras. Están pensadas para niños que ya conocen el alfabeto y comienzan a deletrear, silabear y formar palabras, distinguir entre vocales y consonantes o identificar las letras al verlas escritas o escucharlas.

- La grafomotricidad (movimiento gráfico realizado con la mano al escribir) el reconocimiento de la grafía de las letras. Lo habitual es que en ellas se explique

\subsection{Aplicaciones inclusivas}

La educación inclusiva es aquella que defiende que la escuela debe hacer frente a todas las necesidades de los alumnos independientemente de sus características y circunstancias, y por lo tanto incorporar en las aulas alumnos con discapacidad.

Siguiendo esta idea las apps inclusivas serían ilustraciones de animales u objetos sencillos y reconocibles y en ellas la gamificación y las recompensas juegan un papel esencial. En esta categoría se recomiendan: Drawanimal, ABCKit, Dic Dic, Aprende a leer, MiniU Alfabeto Zoo, Aprende el alfabeto jugando y el Abecedario (Ver Anexo 2).

cómo realizar un determinado trazo con distintas tipologías de letras y que se combinen los ejercicios de escritura.

En muchas aplicaciones de este tipo se trabajan indistintamente los dos aprendizajes, destacamos ABCKit for 5, Freefall, Spelling Spanish, Alfabeto y palabras, Ludiletras, ABC Spanish Reading Magic, Yo escribo (lite) o su versión de pago Comenzando a escribir, El Sonido de las Letras y Dibuja el abecedario.

aquellas que nos permiten adaptar la lectura o el aprendizaje a las capacidades lectoras de cada niño y son especialmente útiles para niños con dificultades cognitivas, dislexia, autismo, dificultades de visión, TGD (Trastorno Generalizado del Desarrollo) o cualquier otra necesidad especial. 
En realidad todas las aplicaciones que sonidos cotidianos y reconocibles. Entre ellas nos permiten adaptar y personalizar los estarían las dirigidas a la enseñanza de la contenidos podrían incluirse en esta categoría, lectura y la escritura, pero también cuentos pero además en este caso, los desarrollos se y otras historias cuyos protagonistas son caracterizan por no enfatizar los errores, niños con las mismas dificultades como, por resaltar los aciertos y proponer objetos y ejemplo, Piruletras o las Letras y yo.

\subsection{Storytelling}

Este término hace referencia a las este tipo de desarrollos les permitirá practicar aplicaciones que permiten la creación de la escritura, la gramática, la ortografía y cuentos personalizados, aunque no sean por supuesto la creatividad, entre las que se necesariamente educativas. La utilización de encuentran Catapum, o CreaAPPcuentos.

\subsection{Jugar con las letras y las palabras}

En este apartado se incluyen aquellas que sin estar pensadas para el aprendizaje pueden ayudar a profundizar y afianzar la lectura y la escritura. Hemos de señalar no obstante que la mayoría de las aplicaciones educativas

\subsection{Practicar, comprender y disfrutar}

La lectura no termina con la decodificación de las palabras y por lo tanto su enseñanza no concluye con el dominio del código alfabético. Los aspectos relacionados con la comprensión lectora deben seguir trabajándose durante toda la etapa escolar, por lo que se deben incorporar también todas aquellas aplicaciones de lectura que pueden servir no solo para el de lectoescritura incluyen el juego como parte integrante de su metodología para aumentar la motivación del alumno. Como ejemplo de esta categoría estarían los crucigramas, sopas de letras, el ahorcado...

aprendizaje, sino también para practicar, para trabajar la comprensión lectora y por supuesto para disfrutar.

Son demasiadas las que habría que incluir en este apartado, Play Tales, Joy Tales, Auca, Mis cuentitos...Sus especificaciones requieren un estudio pormenorizado que será objeto de un próximo trabajo sobre el tema. 


\section{Conclusión}

Como afirma Marchesi (2009) el desafío acelerado de la sociedad de la información está suponiendo retos, impensables hace unos años, para la educación y el aprendizaje. Tal vez lo más relevante sea que nos encontramos con una nueva generación de aprendices que no han tenido que acceder a las nuevas tecnologías, sino que han nacido con ellas y que se enfrentan al conocimiento desde postulados diferentes a los del pasado. Ello supone un desafío enorme para los profesores, la mayoría de ellos inmigrantes digitales, para las escuelas, para los responsables educativos y para los gestores de las políticas públicas relacionadas con la innovación, la tecnología, la ciencia y la educación.

Las tabletas y las aplicaciones cada vez estarán más presentes en la vida cotidiana de los niños incluyendo las aulas. Por ello es imprescindible que los docentes mantengan una actitud abierta ante la utilización de estas herramientas y especialmente formarse en su uso para poder desarrollar actividades didácticas adecuadas.

Conocer dispositivos, productos, recursos para la selección, contenidos, etc. es fundamental para poder aprovechar al máximo las potencialidades de la tecnología. Y todo ello sin olvidarnos nunca de que una tableta puede sustituir a un libro o un cuaderno pero jamás a un MAESTRO, que es el que enseña, corrige y anima al niño en el proceso de de aprendizaje.

En realidad, el uso de las tabletas en el aula es un tema sobre el que hay opiniones enfrentadas, mientras que, como ya hemos visto, cada vez son más las experiencias que parecen avalar su uso como herramienta útil en el contexto escolar, otras alertan de $\mathrm{su}$ excesivo protagonismo (Chatzlopoulos, 2013) mientras algunas consideran que falta mucho para llegar a demostrar los beneficios pedagógicos de las tecnologías (Livingston, 2011).

\section{Referencias}

Chatzlopoulos. N. (2013). iPads in the Chiong, C.; Ree, J.; Takeuchi, L; Erickson, classroom: the right questions you should I. (2012). Print books vs. e-books: Comparing ask. Eudemic. Connecting Education $\&$ parent-child co-reading on print, basic, Technology. http://www.edudemic.com/ and enhanced e-book platforms. The ipads-in-the-classroom-the-right-questions- Joan Ganz Cooney Center http://www. you-should-ask/

joanganzcooneycenter.org/wp-content/ uploads/2012/07/jgcc_ebooks_quickreport.pdf 
Guillem, J. A.; Celaya, J. (2014). Apps en España, PROTEGELES. Disponible en: educativas: nuevas formas de acceso al http://www.diainternetsegura.es/descargas/ conocimiento. Madrid, Dosdoce. estudio_movil_smartphones_tablets_v2c. pdf.

Ferrerio, E.; Teberosky, A. (1979). Los sistemas de escritura en el desarrollo del Metainvestigación Dim-Edu 2013-2014 sobre niño. México; Siglo XXI. el uso educativo de las tabletas digitales. Portal de las tabletas digitales y los libros Informe Tab: estudio sobre el comportamiento de texto digitales. Disponible en: http:// de los usuarios de Tablet en España (2013). Logroño: Universidad Internacional de La Rioja. Disponible en: http://www. tabinnovation.com/informetab/

peremarques.net/tabletasportada.htm

Northrop, L.; Killen, E. (2013). A framework for using iPads to build early literacy skills. The Reading Teacher, 66(7), 531-537. http://

Livingston, S. (2011) Critical reflections on dx.doi.org/10.1002/TRTR.1155 the benefits of ICT in education. Oxford Review of Education, 38(1), 9-24. http:// dx.doi.org/10.1080/03054985.2011.577938

Nuevos modelos de negocio en la era digital. Dosdoce.com con el patrocinio de CEDRO con motivo del lanzamiento de su plataforma Marchesi, A. Preámbulo. En Carneiro, Conlicencia.com. 2014. Madrid. Disponible R., Toscano, J. C., \& Díaz, T. (2009). Los desafíos de las TIC para el cambio educativo. Fundación Santillana. España.

en: http://www.dosdoce.com/upload/ ficheros/noticias/201502/nuevos_modelos_ negocio.pdf.

Martín, S. (2011). La escuela 2.0. Panorama Perspectivas 2014: Tecnología y Pedagogía Actual de la situación del Programa. X (2014). Barcelona: Planeta. Disponible en: Seminario de del Para el $\mathrm{X}$ seminario del Consejo Escolar de Castilla y León. Red http://www.aulaplaneta.com/descargas/ aulaPlaneta_Perspectivas-2014.pdf.

XXI: una puerta hacia la educación del futuro. Disponible en: http://scopeo.usal. es/ponencias-comunicaciones/\#sthash. Z9esUESt.dpuf.

Prensky, M. (2001). Nativos digitales, inmigrantes digitales. On the horizon, 9 (5). http://files.educunab.webnode. $\mathrm{cl} / 200000062$ - 5 aba 35 bb 22 / NativosMenores de Edad y Conectividad Móvil en España: Tablets y Smartphones (2014). Centro de Seguridad en Internet para los Menores

digitales-parte1.pdf http://dx.doi. org/10.1108/10748120110424816

Proyecto e-book y educación. Dedos: tabletas 
digitales en el aula. CITA. Fundación uploads/2012/01/ilearnii.pdf

Germán Sánchez Ruipérez. http://www.

territorioebook.com/includes/descarga. Tonucci: "El alimento de la escuela debería php?id=../recursos ser la experiencia de los niños". Tiching: el blog de educación y TIC. [12-09-2013].

Shuler, C. (2012) iLearn II Analisis of http://blog.tiching.com/francesco-tonucciEducation Category of Apple's App Store el-alimento-de-la-escuela-deberia-ser-laNew York; Joan Ganz Center and Sesame experiencia-de-los-ninos/

Workshop. Disponible en http://www. joanganzcooneycenter.org/wp-content/

\section{Anexos}

\subsection{Fuentes y recursos sobre aplicaciones educativas}

\begin{tabular}{|c|c|}
\hline Androides en el aula & https://android1x1.wordpress.com \\
\hline $\begin{array}{l}\text { A list of All The Best } \\
\text { iPad Apps Teachers } \\
\text { Need }\end{array}$ & $\begin{array}{l}\text { http://www.educatorstechnology.com/2012/12/a-list-of-all-best- } \\
\text { ipad-apps-teachers.html? } \mathrm{m}=1\end{array}$ \\
\hline $\begin{array}{l}\text { Appitic. App list for } \\
\text { education }\end{array}$ & $\begin{array}{l}\text { http://www.appitic.com/index.php/bloom-s/apps-for-bloom-s- } \\
\text { taxonomy }\end{array}$ \\
\hline $\begin{array}{l}\text { Aprendemos con el } \\
\text { iPad }\end{array}$ & http://ipadinfantilausias.blogspot.com.es/ \\
\hline $\begin{array}{l}\text { Apps educativas para } \\
\text { Android }\end{array}$ & http://www.scoop.it/t/apps-educativas-android \\
\hline $\begin{array}{l}\text { Dedos. Tabletas } \\
\text { digitales en el aula }\end{array}$ & http://www.citafgsr.org/educacion/dedos/ \\
\hline Edtechteacher & http://edtechteacher.org/apps/ \\
\hline Eduapps & http://www.eduapps.es \\
\hline Edutablets & $\begin{array}{l}\text { http://www.edutablets.org/ } \\
\text { https://www.pinterest.com/edutablets/ }\end{array}$ \\
\hline Funeducationalapps & http://www.funeducationalapps.com/ \\
\hline
\end{tabular}




\begin{tabular}{|c|c|}
\hline $\begin{array}{l}\text { Guía apps Educación } \\
3.0\end{array}$ & App de iOS (1,99 euros) realizada por la revista Educación 3.0 \\
\hline Innevery Crea & http://ineverycrea.net/comunidad/ineverycrea \\
\hline Infantil apps & $\begin{array}{l}\text { https://sites.google.com/a/genmagic.net/infantilapps/especial- } \\
\text { tablets }\end{array}$ \\
\hline Ipadízate & http://www.ipadizate.es/ \\
\hline $\begin{array}{l}\text { iPad Multimedia Tools } \\
\text { for creativity }\end{array}$ & https://sites.google.com/site/ipadmultimediatools/home \\
\hline $\begin{array}{l}\text { Las mejores } \\
\text { aplicaciones educativas } \\
\text { en Android }\end{array}$ & $\begin{array}{l}\text { http://recursostic.educacion.es/observatorio/ } \\
\text { web/es/software/software-educativo/1070-las- } \\
\text { mejores-aplicaciones-educativas-para-android?_- } \\
\text { c=XXrC1bmRgIRTE58H69sQ0T7JKKuBrZY0yAaLBuy2mDA }\end{array}$ \\
\hline Proyecto Guappis & http://proyectoguappis.blogspot.com.es/ \\
\hline Steve Jobs School & http://stevejobsschool.nl/\#intro \\
\hline $\begin{array}{l}\text { Las Tabletas en } \\
\text { educación }\end{array}$ & http://peremarques.net/tabletasportada.htm \\
\hline
\end{tabular}

\subsection{Aplicaciones por categorías}

\begin{tabular}{|c|c|c|c|c|c|c|c|}
\hline & Nombre & Logo & $\begin{array}{l}\text { Sistema } \\
\text { operativo }\end{array}$ & Idiomas & Precio & Edad & Descripción \\
\hline \multirow[t]{3}{*}{$\begin{array}{l}\text { Aprendizaje } \\
\text { del alfabeto }\end{array}$} & Drawanimal & & 4 & $\begin{array}{l}\text { Español, inglés, } \\
\text { francés y } \\
\text { alemán }\end{array}$ & $1.99 €$ & $\begin{array}{l}\text { Hasta } 5 \\
\text { años }\end{array}$ & $\begin{array}{l}\text { Aplicación destinada a aprender el abecedario y relacionar la } \\
\text { grafía y el sonido dibujando y jugando con los animales. } \\
\text { Su originalidad radica en que es el niño el que tiene que } \\
\text { dibujar sobre un papel el animal que empiece por la inicial } \\
\text { correspondiente, utilizando tanto la tablet como un lápiz } \\
\text { digital y un objeto físico como el papel. }\end{array}$ \\
\hline & ABCKit & & & $\begin{array}{l}\text { Español, } \\
\text { catalán e inglés }\end{array}$ & $\begin{array}{l}1.99 € \\
\text { Compras } \\
\text { integradas }\end{array}$ & $\begin{array}{l}\text { Hasta } 5 \\
\text { años }\end{array}$ & $\begin{array}{l}\text { En esta aplicación se utilizan tres tipos de ejercicios: Conoce } \\
\text { que consiste en identificar el sonido de las letras con una } \\
\text { imagen familiar; escucha en el que se oye la fonética d cada } \\
\text { grafía y escribe, en el que los niños escriben con un trazo } \\
\text { guiado tanto en mayúsculas como en minúsculas. } \\
\text { legida en el } 2011 \text { como la tercera mejor aplicación para iPad } \\
\text { por App Store España, tercera mejor app para iPad en el } \\
\text { Rewind } 2011 \text { en España, Laus de Oro a la mejor app en la } \\
\text { categoría de móviles tablets en el } 2012 \text {. }\end{array}$ \\
\hline & Dic Dic & -1) & 3 & $\begin{array}{l}\text { Español, } \\
\text { catalán francés } \\
\text { e inglés }\end{array}$ & $2.99 €$ & 3-6 años & $\begin{array}{l}\text { Aplicación sencilla y muy intuitiva en la que la voz de un } \\
\text { niño, que cambia en cada lengua, pronuncia una palabra } \\
\text { acompañadas de una imagen representativa que el usuario } \\
\text { tiene que escribir correctamente. } \\
\text { El tratamiento de los errores está especialmente cuidado } \\
\text { para que los niños no tengan miedo a equivocarse y va } \\
\text { acompañada de una serie de recompensas que permiten } \\
\text { conseguir un juego extra cuando se llega a un número } \\
\text { determinado de aciertos. Se puede personalizar el nivel de } \\
\text { dificultad, la lengua y determinar el uso de pistas. La }\end{array}$ \\
\hline
\end{tabular}




\begin{tabular}{|c|c|c|c|c|c|c|c|}
\hline & Nombre & Logo & $\begin{array}{l}\text { Sistema } \\
\text { operativo }\end{array}$ & Idiomas & Precio & Edad & Descripción \\
\hline & & & & & & & escritura se realiza a través del teclado del dispositivo. \\
\hline & Aprende a leer & & & $\begin{array}{l}\text { Español, } \\
\text { alemán, chino, } \\
\text { francés, inglés, } \\
\text { japonés, } \\
\text { portugués y } \\
\text { ruso }\end{array}$ & $\begin{array}{l}\text { Android } 0,70 € \\
\text { iOS versión lite } \\
\text { gratuita y } \\
\text { versión de } \\
\text { pago } 0,99 €\end{array}$ & \begin{tabular}{|l} 
Hasta 5 \\
años
\end{tabular} & $\begin{array}{l}\text { Aprende a leer está pensada para que el niño pueda utilizarla } \\
\text { de forma autónoma a través de dos niveles de juego. } \\
\text { El nivel } 1 \text { está dirigido a niños que todavía no conocen las } \\
\text { letras. En él pueden componer palabras escuchando el } \\
\text { nombre de cada letra con la ayuda de pistas tanto visuales } \\
\text { como sonoras. } \\
\text { El nivel } 2 \text { tiene una mayor dificultad está destinado a niños } \\
\text { que ya conocen las letras y deben componer cada palabra } \\
\text { solo con pistas sonoras en el orden correcto de izquierda a } \\
\text { derecha. } \\
\text { En el apartado de adultos es posible compartir y valorar la } \\
\text { aplicación en Twitter o por correo electrónico. } \\
\text { Para poder utilizarla es necesaria la conexión a Internet. }\end{array}$ \\
\hline & $\begin{array}{l}\text { Mini-U. } \\
\text { Alfabeto Zoo }\end{array}$ & 2 & $\sigma$ & $\begin{array}{l}\text { Español, francés } \\
\text { e inglés }\end{array}$ & $2,99 €$ & $\begin{array}{l}\text { Hasta } 5 \\
\text { años }\end{array}$ & $\begin{array}{l}\text { Mini-U es una app para el aprendizaje de las letras mediante } \\
\text { la asociación de estas con diferentes animales. } \\
\text { Está formada por } 52 \text { tarjetas y en cada una de las pantallas } \\
\text { hay que hacer una actividad con una animal diferente que } \\
\text { representa la letra. Cuenta con dos modalidades: estudio, en } \\
\text { el que la letra aparece en la pantalla junto a la imagen de un } \\
\text { animal que empiece con ella, y acertijo en la que el niño debe } \\
\text { elegir la letra por la que empiece el animal representado. } \\
\text { En el apartado de adultos se puede activar o desactivar la } \\
\text { música y la voz. } \\
\text { No es posible cambiar el idioma dentro de app, cada uno se } \\
\text { adquiere por separado. }\end{array}$ \\
\hline & $\begin{array}{l}\text { Aprende el } \\
\text { alfabeto } \\
\text { jugando }\end{array}$ & $\therefore \frac{\mathrm{A}}{\mathrm{A}}$ & 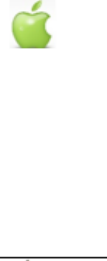 & $\begin{array}{l}\text { Español, } \\
\text { alemán, francés, } \\
\text { inglés, italiano } \\
\text { y neerlandés }\end{array}$ & $\begin{array}{l}1,99 € \\
\text { Compras } \\
\text { integradas }\end{array}$ & $\begin{array}{l}\text { Hasta } 5 \\
\text { años }\end{array}$ & $\begin{array}{l}\text { La aplicación está formada por un conjunto de } 6 \text { juegos } \\
\text { diferentes: escucha la pronunciación, encuentra la letra } \\
\text { anterior y la siguiente, encuentra las dos letras siguientes, } \\
\text { encuentra las dos letras anteriores, coloca las letras en el } \\
\text { orden correcto (de la A la Z) y coloca las letras en el orden } \\
\text { inverso. } \\
\text { Disponible en bundle con Mis primeros puzles: el alfabeto, } \\
\text { Dibuja el abecedario, el ahorcado HD y My first words por } \\
4,99 € .\end{array}$ \\
\hline & El abecedario & 23 & & Español & $\begin{array}{l}\text { Gratuita en } \\
\text { Android, } 0,99 \\
€ \\
\text { para iOS }\end{array}$ & $\begin{array}{l}\text { Hasta } 5 \\
\text { años }\end{array}$ & $\begin{array}{l}\text { Aplicación basada en el juego formada por varios módulos: } \\
\text { Aprender en el que una guacamaya va mostrando las letras } \\
\text { del abecedario para que el niño las conozca y las memorice. } \\
\text { Jugar en donde el mismo personaje menciona una letra y el } \\
\text { niño debe escoger entre varias opciones. } \\
\text { Vocabulario, un carrusel en } 3 \mathrm{D} \text { con } 27 \text { tarjetas interactivas } \\
\text { formadas por palabras y su pronunciación, aunque existe la } \\
\text { posibilidad de eliminar el audio. } \\
\text { Escribir en el que el niño puede seleccionar una letra para } \\
\text { comprobar su grafia y su fonética en una pizarra tradicional. }\end{array}$ \\
\hline $\begin{array}{l}\text { Aprender a } \\
\text { leer y escribir }\end{array}$ & ABCKIT for 5 & for & 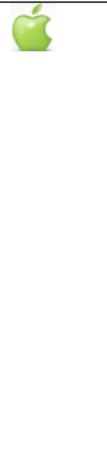 & Español e inglés & $2,99 €$ & 6-8 años & $\begin{array}{l}\text { Desarrollada por Arquinauta y basada en el sistema de } \\
\text { aprendizaje Montessori, los niños aprenden a identificar los } \\
\text { fonemas de cada letra con su grafía, componer y deletrear } \\
\text { fonéticamente palabras, escribir a partir de fonemas y } \\
\text { desarrollar la psicomotricidad. } \\
\text { Incluye cuatro tipos de actividades: } \\
\text { Empareja. Se identifican las letras de forma sonora y visual } \\
\text { para componer palabras. } \\
\text { Ordena. Formar palabras según se van deletreando cada uno } \\
\text { de los fonemas que lo componen. } \\
\text { Completa. Jugar a formar una palabra simplemente } \\
\text { escuchándola } \\
\text { Escribe. Los niños escriben una palabra a partir de cada una } \\
\text { de las letras que la componen. } \\
\text { Disponible en Bundle con ABCKit por } 3,99 € .\end{array}$ \\
\hline
\end{tabular}




\begin{tabular}{|c|c|c|c|c|c|c|}
\hline Nombre & Logo & $\begin{array}{l}\text { Sistema } \\
\text { operativo }\end{array}$ & Idiomas & Precio & Edad & Descripción \\
\hline $\begin{array}{l}\text { Freefall } \\
\text { Spelling } \\
\text { Spanish }\end{array}$ & SPants: & & Español e inglés & $1,79 €$ & 5-6 años & $\begin{array}{l}\text { En esta aplicación se incluyen tres modos: } \\
\text { Freefall: las letras caen por la pantalla a modo de cascada y } \\
\text { el niño tiene que atraparlas y ordenarlas de forma correcta. } \\
\text { Tipo: es necesario buscar en el teclado virtual las letras que } \\
\text { componen las palabras. } \\
\text { Reparto: El objetivo es arrastrar las letras, diseminadas por } \\
\text { el centro de la pantalla, y formar la palabra en las casillas } \\
\text { dispuestas en la parte inferior. } \\
\text { Los juegos incluyen varios ritmos y niveles de juego, se } \\
\text { puede activar y desactivar aquellas secciones que no } \\
\text { utilicemos y dispone de un apartado de recompensas con el } \\
\text { que podemos jugar dando de comer a los animales que } \\
\text { hayamos ganados. } \\
\text { Las palabras se acompañan de una pista visual y un audio } \\
\text { que nos permite escuchar ese término tanto en español como } \\
\text { en inglés. }\end{array}$ \\
\hline $\begin{array}{l}\text { Alfabeto y } \\
\text { palabras }\end{array}$ & & 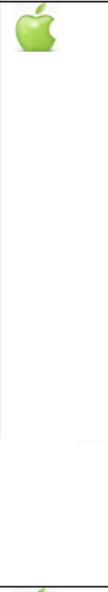 & Español & $\begin{array}{l}\text { Versión lite } \\
\text { gratuita. } \\
\text { Versión de } \\
\text { pago } 2,99 € \\
\text { Compras } \\
\text { integradas }\end{array}$ & 3-5 años & $\begin{array}{l}\text { Aplicación que divide el aprendizaje de la lectoescritura en } \\
\text { tres tipos de juegos con diferentes niveles de dificultad. } \\
\text { El juego de las Letras está protagonizado por unos ositos } \\
\text { polares cuya misión es el aprendizaje de las letras } \\
\text { mayúsculas y minúsculas, como se escriben y se pronuncian, } \\
\text { asociándolas a palabras comunes y con tres niveles de juego. } \\
\text { El juego de las sílabas está guiado por unos pingüinos } \\
\text { encargados de enseñarte las sílabas uniendo las vocales y las } \\
\text { consonantes. Todos los ejercicios van acompañados de } \\
\text { tiempo y la posibilidad de dos jugadores. } \\
\text { En el juego de las palabras unas focas ayudan al niño a } \\
\text { aprender a colocar las letras en el orden correcto y a } \\
\text { deleltrearlas. } \\
\text { Dispone de una Zona de padres a través de la cual se puede } \\
\text { combrobar el progreso del niño pero es necesario descargarlo } \\
\text { previamente. } \\
\text { Esta app Forma parte del programa Aprendes, un programa } \\
\text { educativo para niños en edad preescolar diseñado para } \\
\text { dispositivos táctiles y basado en el juego } \\
\text { (http://www.ilearnwith.com//?llang=es). }\end{array}$ \\
\hline Ludiletras & की & 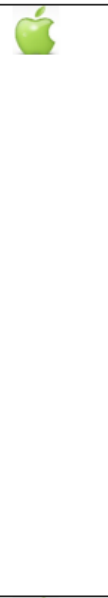 & Español & $1,99 €$ & 3-6 años & $\begin{array}{l}\text { Ludiletras es una app de lectoescritura desarrollada por los } \\
\text { pedagogos del Colegio Montserrat de Barcelona y la editorial } \\
\text { Tekman Books, cuyo objetivo es el aprendizaje de la lectura } \\
\text { y la escritura. } \\
\text { Es una aplicación que, como el resto, utiliza el juego como } \\
\text { herramienta para el aprendizaje. En este caso cada letra se } \\
\text { asocia a un gesto y a una palabra facilitando que } \\
\text { identifiquen el número de letras que tiene una palabra } \\
\text { pasando de forma natural de la fase silábica a la alfabética. } \\
\text { El abecedario, para aprender que gesto corresponde a cada } \\
\text { fonema. } \\
\text { Palabras y gestos. En este caso se trabaja la lectura y el } \\
\text { reconocimiento de la grafía de modo que el niño tiene que } \\
\text { seleccionar la letra y la palabra representadas por ese gesto. } \\
\text { Gesto con palabra. El niño tiene que identificar que gesto es } \\
\text { y escribir la palabra asociada a él arrastrando las letras. } \\
\text { Incluye tres niveles de juego. } \\
\text { También incluye la escritura de letras y palabras mediante la } \\
\text { letra escolar y cuenta con una serie de personajes que van } \\
\text { acompañando al niño durante todo el proceso. }\end{array}$ \\
\hline $\begin{array}{l}\text { ABC Spanish } \\
\text { Reading Magic }\end{array}$ & SEAT & 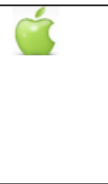 & Español e inglés & Gratuita & 4-6 años & $\begin{array}{l}\text { Está estructurada en tres tipos de actividades para aprender } \\
\text { las letras atendiendo a su fonética. } \\
\text { Juntar sonidos, formada por varias casillas vacías una } \\
\text { principal en la parte superior, tras la cual se esconde la } \\
\text { imagen protagonista, y otras más pequeñas que corresponden } \\
\text { a los sonidos que componen la palabra que debemos adivinar. }\end{array}$ \\
\hline
\end{tabular}




\begin{tabular}{|c|c|c|c|c|c|c|c|}
\hline & Nombre & Logo & $\begin{array}{l}\text { Sistema } \\
\text { operativo }\end{array}$ & Idiomas & Precio & Edad & Descripción \\
\hline & & & & & & & $\begin{array}{l}\text { Separar sonidos: sigue el mismo sistema pero en este caso la } \\
\text { imagen aparece a la vista y el niño tiene que segmentar los } \\
\text { sonidos individuales y emitirlos después. } \\
\text { Lectura: en este caso la imagen está oculta y aparece la } \\
\text { representación gráfica de la palabra que el niño debe leer. } \\
\text { Para comprobar si su lectura es correcta, debe descubrir la } \\
\text { imagen que está detrás de la casilla vacía. } \\
\text { Los tres modos de juego cuentan con una función de audio } \\
\text { que segmenta los sonidos y verbaliza la palabra protagonista } \\
\text { y se puede elegir el número de letras por palabra. }\end{array}$ \\
\hline & $\begin{array}{l}\text { Yo escribo } \\
\text { (lite) } \\
\text { Comenzando a } \\
\text { escribir (de } \\
\text { pago) }\end{array}$ & & $\tilde{\sigma}$ & Español, inglés & $\begin{array}{l}\text { Versión lite } \\
\text { gratuita. } \\
\text { Versión } \\
\text { completa } \\
1,99 €\end{array}$ & & $\begin{array}{l}\text { Yo escribo tiene como objetivo iniciar a los niños en la } \\
\text { escritura formal. En ella, cada una de las vocales se } \\
\text { acompaña de su sonido y una ilustración para que relacionen } \\
\text { cada sonido con una letra y cada letra con un objeto. } \\
\text { Además pueden practicar escribiendo las vocales tanto en } \\
\text { letra de imprenta como manuscrita en diferentes colores, } \\
\text { imprimir, enviar y guardar. } \\
\text { Ofrece también la posibilidad de aprender las palabras que } \\
\text { comienzan con la misma vocal en inglés. }\end{array}$ \\
\hline & $\begin{array}{l}\text { El sonido de } \\
\text { las letras }\end{array}$ & & 6 & $\begin{array}{l}\text { Español, francés, } \\
\text { inglés e italiano }\end{array}$ & $4,99 €$ & $3-7$ & $\begin{array}{l}\text { Esta app basada en la metodología Montessori, incluye } \\
\text { algunas actividades en las que el niño tiene que reconocer la } \\
\text { grafía de una letra con un sonido o con el de una palabra } \\
\text { que contenga esa letra. Incluye una locución que va guiando } \\
\text { al usuario en el manejo de cada uno de los apartados y es } \\
\text { posible personalizar el cuaderno con una foto y el nombre. } \\
\text { Está formada por cuatro secciones: } \\
\text { La caja de los sonidos para la primera etapa en la que el } \\
\text { niño identifica los sonidos con las palabras. } \\
\text { La caja de las letras para la segunda en la que memoriza el } \\
\text { sonido y el trazo de cada letra en cursiva. } \\
\text { Imágenes y letras que le permite asociar las letras y los }\end{array}$ \\
\hline & & & & & & & $\begin{array}{l}\text { sonidos. } \\
\text { El dictado mudo para formar palabras simples con un } \\
\text { alfabeto móvil. } \\
\text { También disponible en dos bundles, Montessori Box ( } 8 \text { app) } \\
\text { por 14,99 € y Montessori } 3-6 \text { años ( } 3 \text { app) por } 6,99 € .\end{array}$ \\
\hline & $\begin{array}{l}\text { Dibuja el } \\
\text { abecedario }\end{array}$ & & & $\begin{array}{l}\text { Español, alemán, } \\
\text { francés, inglés e } \\
\text { italiano }\end{array}$ & $\begin{array}{l}\text { Versión lite } \\
\text { gratuita. } \\
\text { Versión } \\
\text { completa } \\
1,99 € \\
\text { Compras } \\
\text { integradas }\end{array}$ & 4-6 años & $\begin{array}{l}\text { Esta app pretende que los niños aprendan a descubrir cómo } \\
\text { se dibujan las letras y practicar copiando los dibujos. La } \\
\text { aplicación va indicando al usuario los errores y ofrece } \\
\text { recompensas en caso de acierto. } \\
\text { En cada pantalla aparece una letra y los niños deberán usar } \\
\text { las líneas que le guían el trazo para dibujarla igual. } \\
\text { Disponible el bundle "Paquete del alfabeto para niños", junto } \\
\text { con otras } 4 \text { apps por 4,99. }\end{array}$ \\
\hline Inclusivas & Piruletras & & 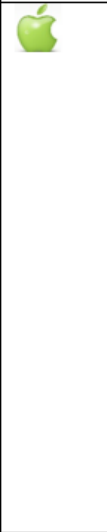 & $\begin{array}{l}\text { Español e } \\
\text { inglés }\end{array}$ & $\begin{array}{l}\text { Versión lite } \\
\text { gratuita. } \\
\text { Desbloqueo } \\
0,99 €\end{array}$ & & $\begin{array}{l}\text { Aplicación dirigida a niños con dislexia, está basada en los } \\
\text { errores de lectura y escritura habituales y en la aplicación } \\
\text { del juegos con cinco niveles de dificultad, inicial, fácili, medio, } \\
\text { dificil y diferentes tipos de ejercicios en función de la } \\
\text { frecuencia de las palabras, longitud, complejidad morfológica } \\
\text { y similitud con otras palabras. } \\
\text { Cada nivel incluye seis tipos de ejercicios con gráficos de la } \\
\text { evolución del aprendizaje por parte de cada niño. } \\
\text { Inserción: se muestra una palabra con una letra en blanco, y } \\
\text { el usuario debe escoger la correcta. } \\
\text { Omisión: la palabra en pantalla contiene una letra de más } \\
\text { que se debe eliminar. } \\
\text { Sustitución: se muestra una palabra con una letra errónea, } \\
\text { que se debe identificar y sustituir por la correcta. } \\
\text { Derivación: se muestran una serie de terminaciones de } \\
\text { palabra y el niño debe escoger qué sufijo es correcto. } \\
\text { Separación: se muestran varias palabras juntas, y el usuario } \\
\text { debe identificar por dónde se separan correctamente. }\end{array}$ \\
\hline
\end{tabular}




\begin{tabular}{|c|c|c|c|c|c|c|c|}
\hline & Nombre & Logo & $\begin{array}{l}\text { Sistema } \\
\text { operativo }\end{array}$ & Idiomas & Precio & Edad & Descripción \\
\hline & & & & & & & $\begin{array}{l}\text { Transposición: se muestran las sílabas o las letras de una } \\
\text { palabra desordenadas, y el usuario debe ordenarlas } \\
\text { correctamente. }\end{array}$ \\
\hline & Las letras y yo & & & $\begin{array}{l}\text { Español, } \\
\text { catalán, } \\
\text { euskera, } \\
\text { gallego, inglés } \\
\text { y francés }\end{array}$ & $1,99 €$ & 5-8 años & $\begin{array}{l}\text { Las letras y yo es en realidad un cuento ilustrado para } \\
\text { entender que es la dislexia en el que una niña, pese a su } \\
\text { esfuerzo, no aprende las letras como sus compañeros. Lo que } \\
\text { pretende la historia es ayudar a otros niños que se } \\
\text { encuentren en situaciones similares. } \\
\text { La adquisición de cada idioma es independiente. }\end{array}$ \\
\hline \multirow[t]{2}{*}{ Stroytelling } & Catapum & & & $\begin{array}{l}\text { Español, } \\
\text { alemán, } \\
\text { catalán, chino, } \\
\text { euskera, } \\
\text { francés, } \\
\text { gallego, inglés, } \\
\text { italiano y ruso }\end{array}$ & $\begin{array}{l}\text { Gratuita } \\
\text { Compras } \\
\text { integradas de } \\
\text { cuentos } 0,99 €\end{array}$ & & $\begin{array}{l}\text { La aplicación de Ediciones Lola Pirindola permite crear tus } \\
\text { propias historias, cambiando el tono de piel de los } \\
\text { personajes, los textos, el tipo de letra, las ilustraciones, } \\
\text { personalizar con la foto y nombre del protagonista, etc. } \\
\text { Los temas de los cuentos son variados y se adaptan a la vida } \\
\text { cotidiana de los niños y a las historias de los cuentos } \\
\text { populares que se pueden cambiar de idioma en la propia } \\
\text { aplicación. }\end{array}$ \\
\hline & CreaPPcuentos & & & & $\begin{array}{l}\text { Gratuita } \\
\text { Compras } \\
\text { integradas de } \\
\text { stickers } 1,99 €\end{array}$ & & $\begin{array}{l}\text { CreAPPcuentos es una aplicación gratuita que permite la } \\
\text { creación de historias a partir de stickers de diferentes temas } \\
\text { con personajes y objetos diferentes, añadir audio, textos en } \\
\text { diferentes tipos y tamaños. } \\
\text { Permite incluir música, personalizar la narración con la voz } \\
\text { del usuario, incluir textos con diferentes tipos de letras, } \\
\text { realizar vídeos con los cuentos y compartirlo etc. } \\
\text { La aplicación gratuita lleva incorporado stickers, piratas en } \\
\text { el caso de la versión para iOS y Caperucita en el caso de } \\
\text { Android. }\end{array}$ \\
\hline \multirow[t]{4}{*}{$\begin{array}{l}\text { Jugar con } \\
\text { letras y } \\
\text { palabras }\end{array}$} & El ahorcado HD & & & $\begin{array}{l}\text { Español, } \\
\text { inglés, alemán, } \\
\text { francés e } \\
\text { italiano }\end{array}$ & $1,99 €$ & 6-8 años & $\begin{array}{l}\text { Otra versión del conocido juego que incluye } 450 \text { palabras y } 5 \\
\text { categorías diferentes: El Oeste, Cuerpo humano y } \\
\text { Vestimenta, Animales, Frutas y Verduras y varios. } \\
\text { Disponible en el bundle "El alfabeto para niños" por } 4,99 € .\end{array}$ \\
\hline & Happi escribe & & & $\begin{array}{l}\text { Español, } \\
\text { alemán, danés, } \\
\text { noruego, } \\
\text { inglés, italiano, } \\
\text { portugués y } \\
\text { sueco }\end{array}$ & $1,99 €$ & 6-8 años & $\begin{array}{l}\text { Happi escribe es un juego de crucigramas que incluye } 60 \\
\text { diferentes divididos en } 5 \text { niveles ( } 12 \text { en cada nivel). Cada } \\
\text { crucigrama consiste en } 4-7 \text { palabras en trenza. El objetivo } \\
\text { del juego es arrastrar las fichas a su lugar indicado en la } \\
\text { trenza con el menor número de errores posibles. } \\
\text { El juego permite cambiar utilizar letras mayúsculas o } \\
\text { minúsculas y el idioma e incluye imágenes y fondos en color. }\end{array}$ \\
\hline & Dominó & $\mathrm{MI}$ & & $\begin{array}{l}\text { Español, } \\
\text { alemán, } \\
\text { francés e inglés }\end{array}$ & $\begin{array}{l}\text { Gratuita para } \\
\text { iOS, } 0,99 € \\
\text { para Android }\end{array}$ & $\begin{array}{l}\text { Desde } 6 \\
\text { años }\end{array}$ & $\begin{array}{l}\text { Esta aplicación, diseñada por un logopeda, está destinada a } \\
\text { trabajar el vocabulario, la lectura y las silabas. } \\
\text { El objetivo fundamental es formar palabras con las silabas } \\
\text { propuestas que en cada juego pertenecen a una categoría } \\
\text { diferente. } \\
\text { Dispone de tres niveles de dificultad con más de } 600 \text { palabras } \\
\text { en la versión completa y } 28 \text { categorías. }\end{array}$ \\
\hline & Pop ABC & & & Inglés &, $99 €$ & 3-10 años & $\begin{array}{l}\text { Entretenido juego en el que las protagonistas son las letras } \\
\text { del alfabeto para niños entre } 3 \text { y } 10 \text { con diferentes niveles de } \\
\text { dificultad. } \\
\text { El objetivo, mezcla entre un puzle y el tetris, es unir el } \\
\text { mayor número de letras iguales, la } \mathrm{A} \text { con la } \mathrm{A} \text {, la } \mathrm{B} \text { con la B, } \\
\text { etc. y al conseguirlo se puede escuchar el fonema. Al } \\
\text { conseguir cada una de las letras se van obteniendo puntos. }\end{array}$ \\
\hline
\end{tabular}

\section{Notas}

${ }^{1}$ Así es la nueva generación de nativos digitales: Los niños ya prefieren las tabletas a los libros http://www.puromarketing.com/12/15257/ nueva-generacion-nativos-digitales-ninos-prefieren-tablets.html

${ }^{2}$ Samsung School está compuesta por un software interactivo y dos tipos de dispositivos a elegir por el centro, ordenadores o tablets (http://www.samsung.com/es/business/solutions-services/mobile-solutions/education/samsung-school)

3 Datos extraídos del portal del Instituto Nacional de Estadística correspondientes al año 2014 véase http://www.ine.es/jaxi/menu. do?type $=$ pcaxis\&path $=/$ t25/p450/base $2011 /$ a2014/\&file $=$ pcaxis

${ }^{4}$ Para más información sobre los modelos de negocio digital consultar Nuevos modelos de negocio en la era digital. Dosdoce (2014)

${ }^{5}$ Con una solución MDM, los administradores pueden implantar los dispositivos de forma segura en entornos educativos, configurar y actualizar los ajustes, definir restricciones e instalar aplicaciones.

${ }^{6}$ Estas aplicaciones pueden estar pensadas para ser utilizadas por los niños pero

también existen aplicaciones pensadas para uso docente como Ender Metrics Metrics (disponible tanto para Android como para iOS) una herramienta para analizar el aprendizaje en apps infantiles. Su objetivo principal es analizar qué y cómo aprenden los niños a través de cada app educativa que utilizan. La podríamos definir como el Google Analytics de la educación infantil. Ender Metrics te permite hacer un seguimiento de cómo evoluciona cada usuario, que es lo que el niño aprende, dónde se pierde y que le gusta más hace, ver si cumple el currículum educativo (para niños de 2 a 6 años). http://endermetrics.com/es/ 